fossil hominid from Yongok-ni (U. J. Jan), and a demonstration of the co-existence at Chon-gok-ni of both large- and small-tool traditions in a single site (K. Bae, Seoul). The large tools from Chon-gok-ni are more like the true hand-axes than most material from east Asia, but the site, like the 'Lower Palaeolithic' industries of Kota Tampan in Malaysia (Z. Majid) and the Patjitanian of Java, seems to be Upper Pleistocene in age.

The conference re-established the importance of China in the overall scheme of later human evolution, and showed that this region is best understood on its own terms rather than by comparing it with western Eurasia. Our understanding of the Chinese palaeolithic record has been hindered by the application of western European parameters of technological development. The widespread use of expedient technologies on poor raw materials such as quartz, the persistence of crude bipolar cores, chopping tools and other 'Lower Palaeolithic' forms into the later Pleistocene, and the absence of well developed blade industries in many regions, all suggest a pattern more common to areas of central and west Africa than to western Europe. We can hope that when 'Peking Man' celebrates his diamond anniversary, the dramatic expansion of research in East Asia will have shed further light on the precise nature of the apparently unique human adaptations that characterize this region, as well as on the relationships between East Asia and Africa, during the emergence of our own species.

Alison S. Brooks is in the Department of Anthropology, George Washington University, Washington DC 20052, USA. Bernu-d Wood is in the Department of Human Anatomy and Cell Biology, Liverpool University, PO Box 147, Liverpool L69 3BX, UK.

1. Chen, T. \& Yuan, S. Archaeometry 30, 59-76 (1988)

2. Wu, R. \& OIsen, J. W. Palaeoanthropology and Palaeolithic Archaeology in the People's Republic of China (Academic. Orlando, 1985)

3. Wu, R., Wu, X. \& Zhang, S. Early Humankind in China (institute of Vertebrate Paleontology and Paleoanthropology, Beijing, 1989) (in Chinese)

\title{
Photons faster than light?
}

\section{Stephen M. Barnett}

ONE of the cornerstones of twentiethcentury physics is the limiting velocity of the speed of light, $c$. Every undergraduate knows that no particle can travel and no signal can propagate at a velocity greater than $c$. This comfortable and well established phenomenon has now been challenged by Scharnhorst ${ }^{1}$ and Barton ${ }^{2}$. They have uncovered a quantum electrodynamic phenomenon that implies the possible existence of faster-than-light photons.

The source of this surprising development is the mysterious nature of the vacuum as revealed by quantum electrodynamics. It is not an empty nothing but contains randomly fluctuating electromagnetic fields and virtual electronpositron pairs with an infinite zero-point energy. Light propagating through space interacts with the vacuum fields, and observable properties, including the speed of light, are in part determined by this interaction. The effect on the speed of light is due to the absorption of photons to form virtual electron-positron pairs followed by the re-emission of the photon. The particle pairs are very short lived because of the large mismatch between the energy of a visible photon and the rest mass of an electron and a positron. Nevertheless, this process makes a contribution to the observed permittivity of the vacuum $\left(\varepsilon_{0}\right)$ and therefore to the speed of light.

The role of the virtual particle pairs in determining the permittivity of the vacuum is analogous to that of atoms or molecules in determining the relative permittivity of a dielectric material. The light propagating in the material can be absorbed, but in a transparent medium there are no strongly absorbing resonances and the atoms remain in their excited states for only a very short time before re-emitting the light. This absorption and re-emission is responsible for the refractive index of the material and results in the well known reduction of the speed of light.

Scharnhorst and Barton suggest that a modification of the vacuum can produce a change in its permittivity with a resulting change in the speed of light. In particular, the suppression of light-scattering from the virtual electron-positron pairs should cause an increase in the speed of light. One way to change the vacuum is to place two large conducting plates parallel to each other, so as to form a cavity or waveguide (see figure). The vacuum field inside the cavity has to be modified so that the electromagnetic boundary conditions are satisfied. The principal result of this modification is the removal of the zero-point energy due to the suppression of vacuum modes with wavelengths longer than the waveguide-cutoff $\left(\lambda_{0}\right)$. It is this removal of free-space vacuum modes that suppresses the scattering of the light by virtual particle pairs and in theory produces the increase in the speed of light.

The predicted increase in the speed of light is extremely small and only occurs

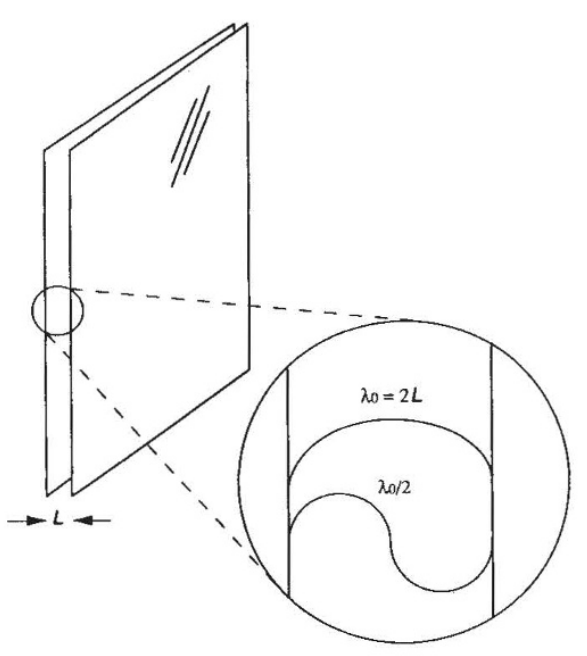

The vacuum field is modified in a waveguide and electromagnetic modes with wavelengths greater than $\lambda_{0}$ are suppressed.

for light propagating perpendicular to the plates. For a plate separation of $1 \mu \mathrm{m}$ the fractional change in the speed of light is about one part in $10^{36}$. This effect is so small that it is far beyond the reach of present experiments, but its significance as a point of principle should not be underestimated. It is worth emphasizing that the increase in the speed of light is a genuine prediction for the propagation velocity. Optical phase and even group velocities can sometimes exceed $c$, but dispersion always ensures that the signal velocity is less than or equal to $c$. In the parallel-plate system described by Scharnhorst and Barton, the dispersive effects are much weaker still than those associated with the apparent increase in the velocity of light; the phase, group and signal velocities will therefore all increase by about the same amount.

The reduction of the vacuum energy density in the region between two parallel conducting and neutral plates has other consequences, most notably the famous Casimir effect. If the plates are moved towards each other then the vacuum energy drops. This means that there is an attractive potential between the plates, the origin of which is purely quantum electrodynamic in nature. The resulting force pulling the plates together has been measured and for a plate separation of $0.1 \mu \mathrm{m}$ has a value of about 13 newtons per square metre of the plate surfaces.

The vacuum is certainly a most mysterious and elusive object that makes itself known by only the most indirect of hints. The suggestion that value of the speed of light is determined by its structure is worthy of serious investigation by theoretical physicists.

Stephen M. Barnett is in the Department of Engineering Science, University of Oxford, Parks Road, Oxford OX1 3PJ, UK.

Scharnhorst, K. Phys. Lett. B236, 354-359 (1990)

2. Barton, G. Phys. Lett. B (in the press). 